\title{
The Effect of Intragastric Administration of Botulinum Toxin Type A on Reducing Adiposity in a Rat Model of Obesity Using Micro-CT and Histological Examinations
}

\author{
Jin-Seok Park ${ }^{1}$, Hong-Mei Zheng ${ }^{2}$, Jae-Min Kim² ${ }^{2}$, Chung Sei Kim ${ }^{3}$, Seok Jeong ${ }^{1,2}$, and Don Haeng Lee Le,2,4 $^{1,2}$ \\ ${ }^{1}$ Digestive Disease Center, Department of Internal Medicine, Inha University School of Medicine, ${ }^{2}$ National Center of Efficacy Evaluation for the \\ Development of Health Products Targeting Digestive Disorders (NCEED), Incheon, ${ }^{3}$ Bio R\&D Center, Daewoong Pharmaceutical, Yongin, and \\ ${ }^{4}$ Utah-Inha DDS \& Advanced Therapeutics Research Center, Incheon, Korea
}

Background/Aims: Botulinum toxin type A (BTX), a longacting inhibitor of muscular contraction in both striated and smooth muscles, is responsible for gastric motility. The aim of this study was to investigate the effects of an endoscopic intragastric BTX injection on weight loss, body fat accumulation, and gastric emptying time. Methods: The BTX group consisted of 15 obese rats in which $20 \mathrm{U}$ of BTX were injected into the gastric antrum. The saline group consisted of 15 obese rats injected with $20 \mathrm{U}$ of saline, and the control group included 10 obese rats that did not receive a surgical intervention. The gastric emptying time, biochemical parameters, and body fat volume were evaluated using microcomputed tomography (micro-CT) and histologic evaluations. Results: The postoperative body weight of the BTX group was significantly lower than those of the other groups $(p<0.001)$ at 6 weeks after the operation. The gastric emptying time (156 \pm 54 minutes) was significantly delayed in the BTX group. The BTX group showed significantly lower lipid levels than the other groups. A reduction in body fat volume was observed in the BTX group using micro-CT and histological evaluations. Conclusions: BTX application to the gastric antrum represents a potentially effective treatment for obesity and may help improve the lipid profile by increasing the gastric emptying time. (Gut Liver 2017;11:798-806)

Key Words: Botulinum toxins; Obesity; Endoscopes

\section{INTRODUCTION}

Obesity is currently one of the major health concerns in developed countries. With more than 1.9 billion people now obese or overweight, the eventual impact of expected adverse events such as diabetes, hypertension, sleep apnea, cardiac failure, reflux, and major orthopedic problems, will take a major toll on public health. ${ }^{1,2}$ Extensive research in the pharmacological treatment of obesity has been conducted. However, to date, there has been no major progress in the development of safe and effective medications for combatting obesity.,4 There may be several reasons for the failure of pharmacologic therapy in obesity, but the lack of early satiety with pharmacological therapy is likely an important factor. To overcome this difficulty, bariatric surgery has been used to treat obesity. ${ }^{5,6}$ However, this surgical treatment requires complicated preparations and postoperative adverse events, such as wound infection. Furthermore, it is possible for surgical treatment to result in death.

Botulinum toxin type A (BTX) has recently been used therapeutically in a vast array of gastrointestinal disorders. The therapeutic effects of BTX occur through temporary chemo-denervation. ${ }^{7}$ Thus far, prior animal and human studies suggest that gastric BTX injections slow gastric emptying, increase satiation, and induce weight loss. ${ }^{8-10}$ However, there is limited information regarding the overall safety and efficacy of BTX therapy. ${ }^{11}$

Therefore, the aim of this study was to evaluate the effects of intragastric BTX injection on treatment of obesity. In addition, we tried to objectify the effect of BTX by applying histologic evaluation, serum biochemistry, and computed tomography (CT), to measure the abdominal and subcutaneous fat volumes.

\section{MATERIALS AND METHODS}

\section{Animal and study design}

Forty-seven-week-old male Sprague Dawley Rats, weighing

\footnotetext{
Correspondence to: Don Haeng Lee

Digestive Disease Center, Department of Internal Medicine, Inha University School of Medicine, 27 Inhang-ro, Jung-gu, Incheon 22332, Korea

Tel: +82-32-890-2548, Fax: +82-32-890-2549, E-mail: inos@inha.ac.kr

Received on November 16, 2016. Revised on January 31, 2017. Accepted on February 7, 2017. Published online July 28, 2017

pISSN 1976-2283 eISSN 2005-1212 https://doi.org/10.5009/gnl16557

Jin-Seok Park and Hong-Mei Zheng contributed equally to this work as first authors.

@ This is an Open Access article distributed under the terms of the Creative Commons Attribution Non-Commercial License (http://creativecommons.org/licenses/by-nc/4.0) which permits unrestricted non-commercial use, distribution, and reproduction in any medium, provided the original work is properly cited.
} 
200 to 220 g were used for this study. After the adaptation period for 1 week, all rats were fed a high-fat diet ad libitum for a 90-day period. After 90 days, the high fat diet-induced obese rats were randomly separated into three groups and underwent surgery, as described below. All experiments were performed in accordance with the institutional guidelines for the care and use of laboratory animals under a license issued by the local ethical committee. Rats were assigned to one of the three groups: BTX group consisted of 15 obese rats injected with $20 \mathrm{U}$ BTX to the gastric antrum; saline group consisted of 15 obese rats injected with $20 \mathrm{U}$ saline solution into the gastric antrum; and control group consisted of 10 obese rats with no surgical intervention.

\section{Botulinum toxin type $A$}

NABOTA $^{\circledR}$ (Daewoong Pharmaceutical, Seoul, Korea), a new BTX originating from the wild-type Clostridium botulinum Hall A, was used in this study. NABOTA ${ }^{\circledR}$ was manufactured by HIPure Technology (Birmingham, UK), confirmed by size exclusion high-performance liquid chromatography analysis showing a single $900 \mathrm{kDa}$ peak (>98\%). ${ }^{12}$ The clinical efficacy and safety of NABOTA ${ }^{\circledR}$ has been established in in vivo studies and clinical trials. $^{12-14}$

\section{Operation procedure}

After overnight fasting, rats were anesthetized with isoflurane (5\% for induction and 2\% to 3\% for maintenance). Under sterile conditions, upper midline laparotomy-approximately $2 \mathrm{~cm}$ in length-was made. For the BTX group, rats were injected with BTX using a 27 gauge syringe. A total of $20 \mathrm{U}$ of the toxin were diluted with $2 \mathrm{~mL}$ saline and injected subserosally into the four cardinal points in the gastric antrum: on the lesser and greater curvature, and on anterior and posterior wall (5 U in each 0.5 $\mathrm{mL}$ injection). The same procedure of anesthesia and laparotomy was applied to the saline group, but injected with saline in the same position of the antrum. The remaining 10 rats did not receive any intervention (control group).

\section{Body weight and food consumption}

The rats were allowed to feed ad libitum the same preoperative high-calorie diet from the first postoperative day to 7 weeks after the operation. The weight of the ingested food was measured at 1-week interval. At the beginning of the study, the body weight of all rats were measured and recorded. The weight gains of the rats were followed by weight measurements at 1-week interval, and the changes were recorded.

\section{Gastric emptying time measurement}

To obtain an image of gastric emptying, a liquid contrast agent (20\% emulsions of barium sulfate) was used. A gastric lavage cannula was inserted via the orogastric route, and a liquid contrast agent was given through the tube. The gastric emptying scan was performed using an X-ray camera. After taking consecutive X-ray radiographs, rats were returned to their cages, and again, allowed free access to food and water. The gastric emptying scan was performed at preoperative 15 days and 30 days after BTX injection, and the each emptying time was compared.

\section{Biochemical parameter analysis of blood}

The blood samples were collected at 3 and 6 weeks after the high fat diet, and every 2 weeks after the injection of gastric BTX. Alanine transaminase (ALT), aspartate transaminase (AST), alkaline phosphate (ALP), albumin, total bilirubin (T-bilirubin), total protein, cholesterol, triglyceride (TG), blood urea nitrogen (BUN), creatinine, high-density lipoprotein (HDL), low-density lipoprotein (LDL), and glucose were determined using blood biochemistry analyzer (7020; Hitachi Ltd., Tokyo, Japan).

\section{Micro-CT (abdominal and subcutaneous fat volume)}

The entire torso of each rat was scanned at an isotropic voxel size of $76 \mu \mathrm{m}$ ( $45 \mathrm{kV}, 177 \mu \mathrm{A}, 200 \mathrm{~ms}$ integration time) with a viva CT 80 scanner (Scanco Medical, Brüttisellen, Switzerland). Two-dimensional gray-scale image slices were reconstructed

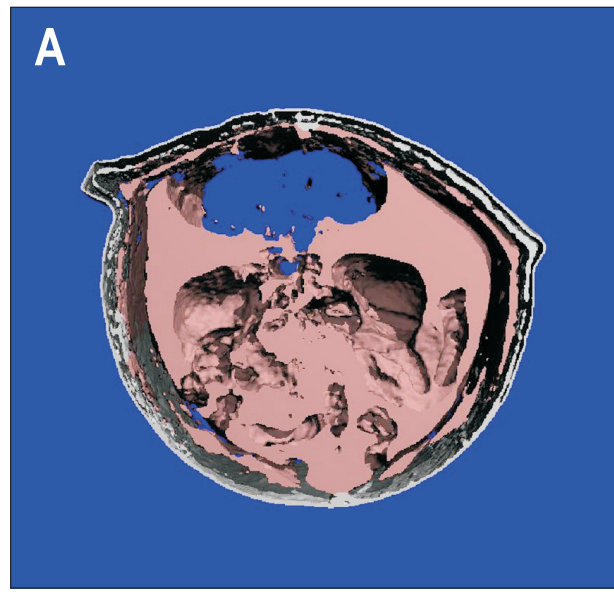

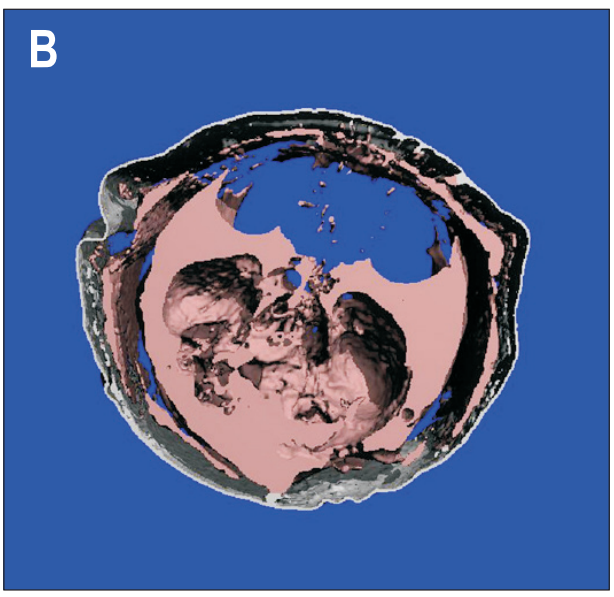

Fig. 1. Representative micro-computed tomography images obtained from two animals in the control (A) and botulinum toxin type A (BTX) (B) groups are shown, with the fatspecific threshold applied. Fat tissue is shown in gray. 
into a three-dimensional tomography. Density values for soft tissue were calibrated from a 5-point linear fit line with mixtures in various ratios of two liquids, ranging from $0.78 \mathrm{mg} / \mathrm{mL}$ (100\% ethanol; Sigma, St. Louis, MO, USA) to $1.26 \mathrm{mg} / \mathrm{mL}(100 \%$ glycerol; J.T. Baker, Phillipsburg, NJ, USA). Image processing language was used to analyze the total fat volume (Fig. 1). The total adipose tissue volume was first evaluated, and then further subdivided into compartments. The fat compartments in the abdominal region were separated and quantified using a custom image processing language script based on the Canny method for edge detection. ${ }^{15}$

\section{Macroscopic and microscopic evaluation}

Euthanasia was performed at 7 weeks after BTX injection. The liver and epididymal and perirenal fat pads were removed, rinsed with phosphate-buffered saline, and then weighted. Liver tissues were cut to a thickness of $4 \mu \mathrm{m}$, deparaffinized, and stained with hematoxylin and eosin (H\&E). Histologic structures were observed by a light microscope. Oil Red 0 staining was performed to evaluate the accumulation of fat droplets in hepatocytes in the liver sections. The ratio of the Oil Red 0 stained area to the total area was determined using an image J software (https://imagej.nih.gov/ij/). To analyze the hepatic lipid content, the lipids were extracted by the Folch method. The contents of total cholesterol (Cholesterol/Cholesteryl Ester Quantitation Assay kit, ab65359; Abcam, Cambridge, MA, USA) and TG (Triglyceride Colorimetric Assay kit, no. 10010303; Cayman Chemical, Ann Arbor, MI, USA) in the hepatic lipid extracts were measured by the commercial kits.

\section{Statistical analysis}

Data are expressed as the mean \pm standard deviation values. Statistical comparisons between the groups were determined by nonparametric Kruskal-Wallis, followed by post hoc MannWhitney U test. The differences were considered statistically significant at a p-value of $<0.05$. Statistical calculations were performed using SPSS software version 19.0 (IBM Corp., Armonk, NY, USA).

\section{RESULTS}

Gastric BTX or saline injection was successfully performed on thirty rats. No technical difficulties or adverse events occurred during the operation. All animals survived until the end of the study.

\section{Effect of BTX on reduction of body weight}

The preoperative and postoperative body weights are shown in Table 1. The initial weights showed no significant difference between the three groups ( $p>0.05$ ). There was a significant increase in body weight in the three groups between the initial day and 90th day. The body weight of the rats which underwent an operation dropped after surgery, but gradually returned to the baseline values, while the rate of weight gain after the weight nadir was significantly different between the BTX group and the saline group $(\mathrm{p}<0.001)$. The postoperative body weight of the BTX group (593.285 $\pm 53.609 \mathrm{~g})$ was significantly reduced than that of the saline $(688.197 \pm 58.140 \mathrm{~g}, \mathrm{p}<0.001)$ and control groups $(734.163 \pm 77.672 \mathrm{~g}, \mathrm{p}<0.001)$ at 6 weeks after the BTX injection (Table 1, Fig. 2). The differences between the saline and control groups were not significant.

\section{Food consumption}

The basal weights of the daily ingested food did not differ among the three groups prior to the operation. Two weeks

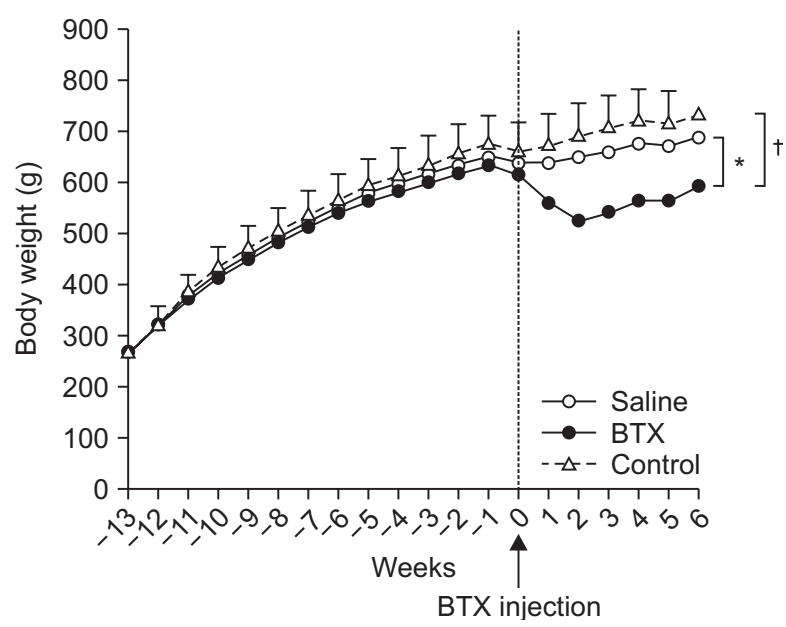

Fig. 2. Time courses of body weight in the control, saline, and botulinum toxin type A (BTX) groups. *A significant difference at $\mathrm{p}<0.01$ level compared to the saline group; ${ }^{\dagger} \mathrm{A}$ significant difference $\mathrm{p}<0.01$ level compared to the control group.

Table 1. Effect of the Intragastric Injection of BTX on Body Weight

\begin{tabular}{lccccccc}
\hline Variable & Before BTX & Week 1 & Week 2 & Week 3 & Week 4 & Week 5 & Week 6 \\
\hline Control & $662.7 \pm 61.7$ & $676.8 \pm 60.9$ & $695.0 \pm 62.5$ & $709.9 \pm 66.4$ & $721.7 \pm 73.4$ & $718.7 \pm 74.4$ & $734.2 \pm 77.7^{*}$ \\
Saline & $640.8 \pm 43.2$ & $639.2 \pm 43.9$ & $649.2 \pm 45.0$ & $659.3 \pm 48.2$ & $675.0 \pm 52.1$ & $673.2 \pm 53.9$ & $688.2 \pm 58.1^{*}$ \\
BTX & $617.7 \pm 51.5$ & $559.4 \pm 47.1^{\dagger}$ & $526.6 \pm 46.4^{\ddagger}$ & $543.3 \pm 47.8^{\dagger}$ & $565.5 \pm 51.7^{\dagger}$ & $567.0 \pm 51.2^{*}$ & $593.3 \pm 53.6$ \\
\hline
\end{tabular}

Data are presented as mean \pm SD.

BTX, botulinum toxin type A.

Compared with before the BTX treatment; ${ }^{*} \mathrm{p}<0.05,{ }^{\dagger} \mathrm{p}<0.01,{ }^{\dagger} \mathrm{p}<0.001$. 
after the surgery, dietary intake in the BTX and saline groups was significantly smaller than the control group $(\mathrm{p}<0.001)$, and subsequently increased in both groups, although the BTX group showed a more pronounced and longer-lasting reduced intake compared with the saline group. To recover the basal amount of daily food intake, the saline group required 3 weeks, but the BTX group needed 4 weeks (Fig. 3).

\section{Effect of BTX on gastric emptying time}

There was no significant difference in gastric emptying time between the three groups preoperatively. There was no significant difference between the gastric emptying times on day of the operation and postoperative day 30 in the control group (114 \pm 48 minutes vs $114 \pm 36$ minutes) and the saline group (108 \pm 30 minutes vs $108 \pm 48$ minutes). Conversely, the gastric

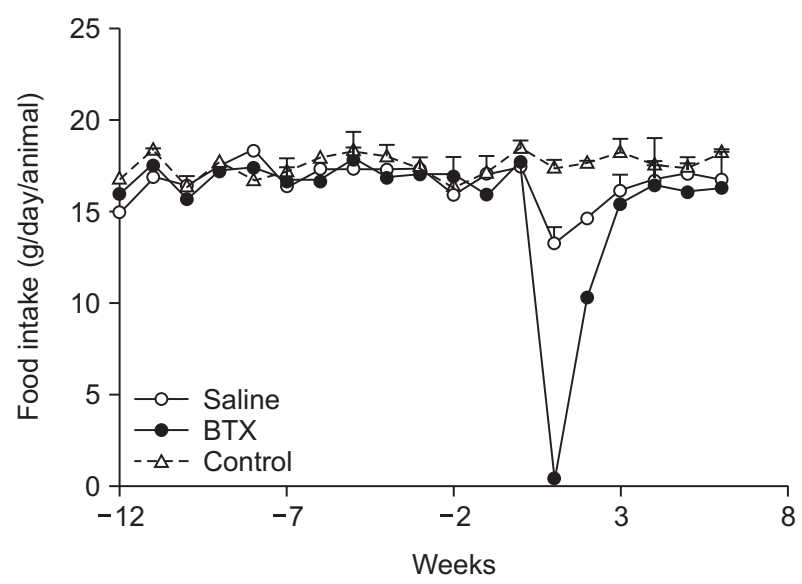

Fig. 3. Time courses of food intake in the control, saline, and botulinum toxin type A (BTX) groups. emptying time in the BTX group was significantly delayed after the operation (102 \pm 42 minutes vs $156 \pm 54$ minutes). With respect to the result from a comparison between the saline group and BTX group, the gastric emptying time was significantly prolonged in the BTX group than in the saline group at postoperative 1 month $(\mathrm{p}<0.05)$ (Fig. 4).

\section{Effect of BTX on lipid parameters in serum}

Fig. 5 shows the values of the serum lipid parameters in all three groups. All groups showed increased serum lipid profile after a high-fat diet, and the basal lipid profiles on the operation day were not significantly different between the groups. The BTX group showed a significantly lower levels of total cholesterol (BTX, 135.0 $\pm 10.6 \mathrm{mg} / \mathrm{kg}$; control, $151.0 \pm 18.3 \mathrm{mg} / \mathrm{kg}$;

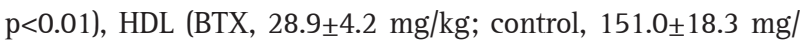
$\mathrm{kg} ; \mathrm{p}<0.05$ ), and LDL (BTX, 9.3 $\pm 3.1 \mathrm{mg} / \mathrm{kg}$; control, 14.6 \pm 4.1 $\mathrm{mg} / \mathrm{kg} ; \mathrm{p}<0.01)$ compared with the control group at postoperative 4 weeks.

To evaluate the potential toxic effects of BTX on the hepatic and renal functions, we measured the serum ALT, AST, Tbilirubin, ALP, BUN, and creatinine levels in all rats. The levels of hematologic markers were not significantly changed in the BTX groups compared with the levels in the saline and control groups (Table 2).

\section{Effect of BTX on body fat volume}

The total fat volume was markedly reduced in the BTX group $\left(33,998.13 \pm 10,554.09 \mathrm{~mm}^{3}\right)$ compared with the control group $\left(59,109.25 \pm 18,064.71 \mathrm{~mm}^{3}, \mathrm{p}<0.001\right)$ (Fig. 6) at 4 weeks after the BTX injection on micro-CT evaluation, and the tendency of reduction was maintained at the end of experiment (BTX, $41,583.08 \pm 10,432.88 \mathrm{~mm}^{3}$; control, 61,196.56 $\pm 18,200.03 \mathrm{~mm}^{3}$;
A

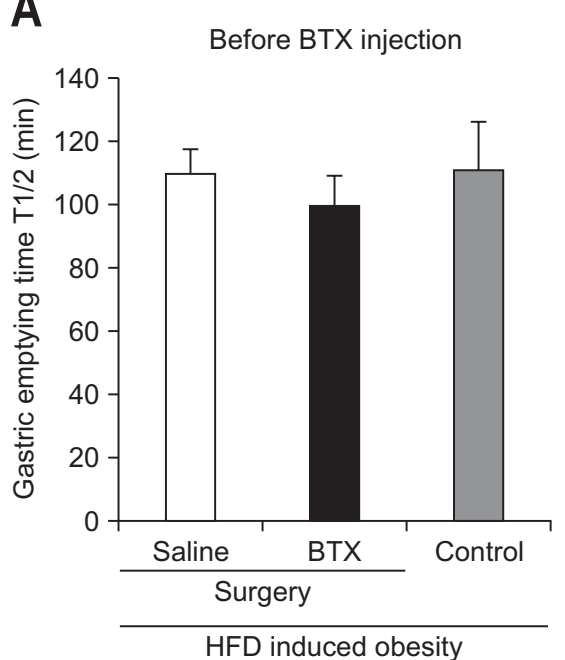

B

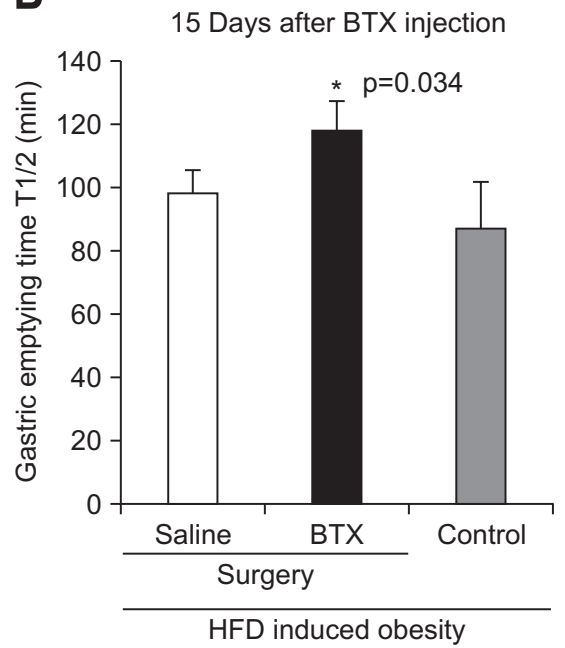

C

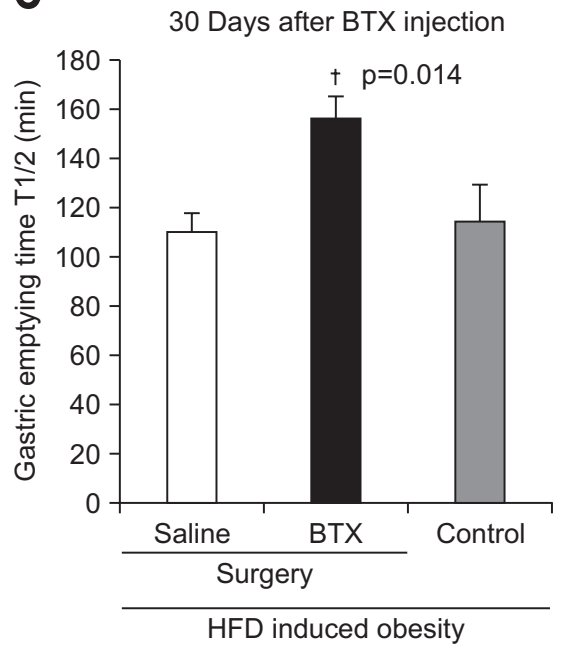

Fig. 4. Gastric emptying time values of the three groups on surgery day (A), day 15 (B), and day 30 (C) after surgery.

BTX, botulinum toxin type A; HFD, high fat diet. ${ }^{*}$ A significant difference $p<0.05$ level compared to the control group; ${ }^{\dagger}$ A significant difference at $\mathrm{p}<0.05$ level compared to the saline group. 
A

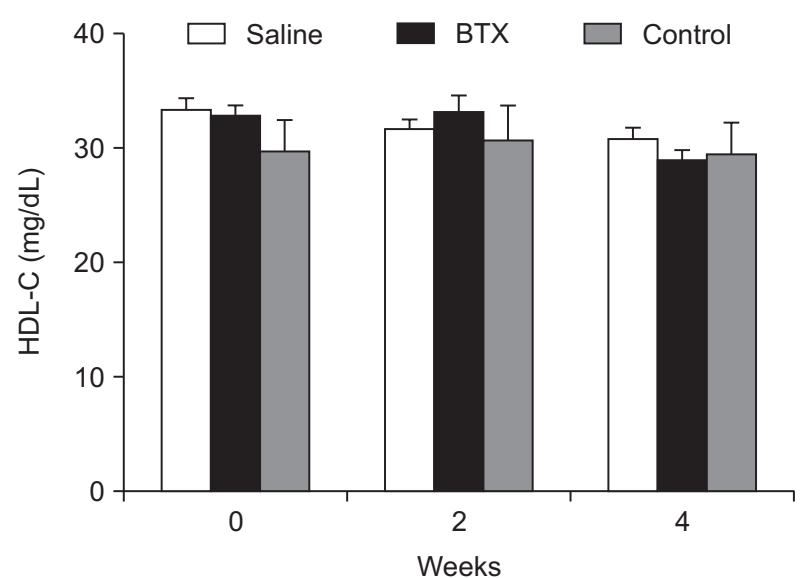

C

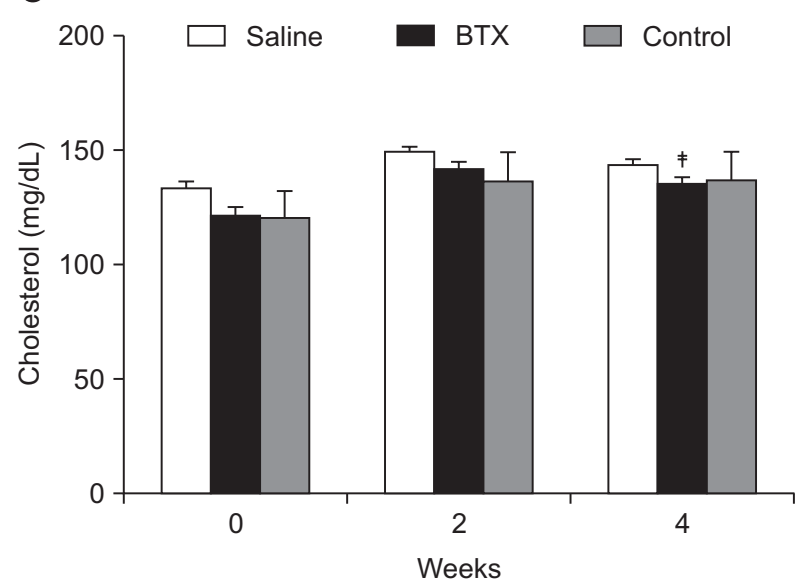

B

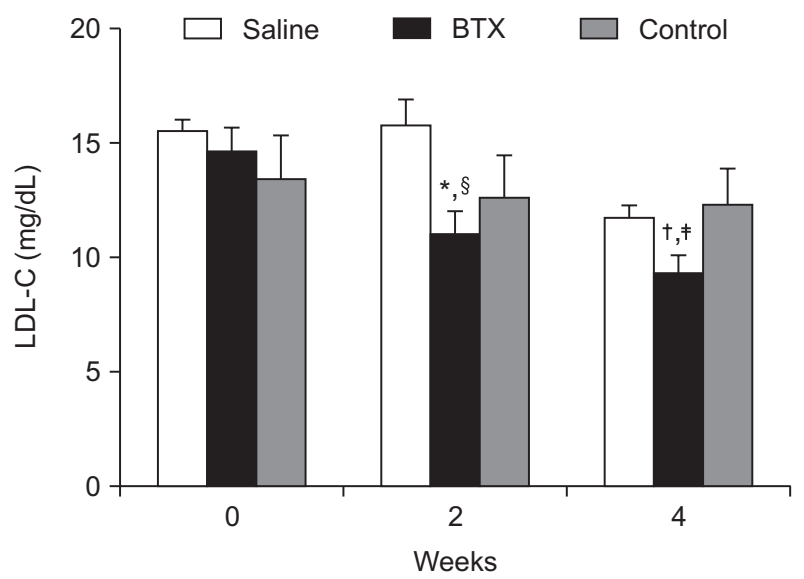

D

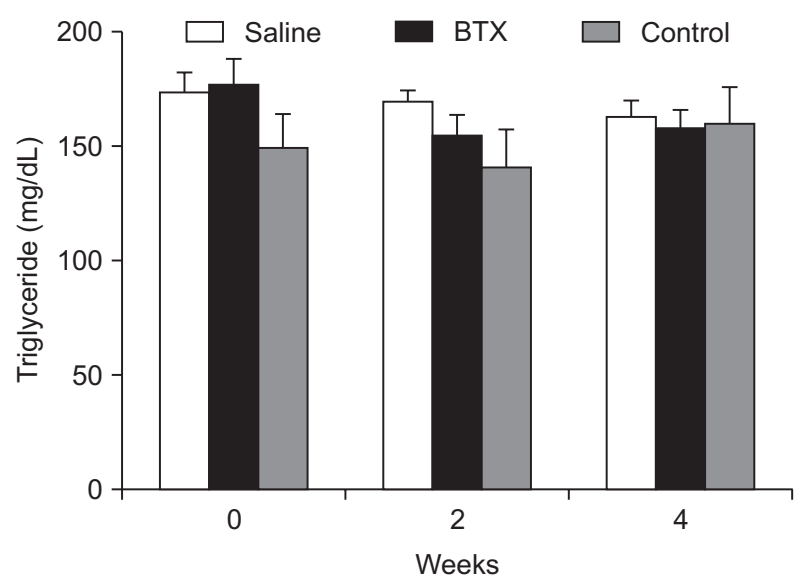

Fig. 5. The values of serum lipid parameters in the three groups are shown; high-density lipoprotein (HDL, A), low-density lipoprotein (LDL, B), total cholesterol (C), and triglyceride (D). The botulinum toxin type A (BTX) group showed a significantly lower total cholesterol level ( $<<0.05)$ and LDL level $(\mathrm{p}<0.01)$ than the control group at 4 weeks after surgery.

A significant difference at ${ }^{*} \mathrm{p}<0.01,{ }^{\dagger} \mathrm{p}<0.05$ level compared to the saline group; A significant difference ${ }^{\ddagger} \mathrm{p}<0.01,{ }^{\mathrm{s}} \mathrm{p}<0.05$ level compared to the control group.

Table 2. Changes in the Levels of Serum Biomarker in the Control, Saline, and BTX Groups after the Operation

\begin{tabular}{|c|c|c|c|c|c|c|}
\hline \multirow{2}{*}{ Test } & \multicolumn{2}{|c|}{ Saline } & \multicolumn{2}{|c|}{ BTX } & \multicolumn{2}{|c|}{ Control } \\
\hline & Before OP & 4 wk after OP & Before OP & 4 wk after OP & Before OP & 4 wk after OP \\
\hline AST (GOT), U/L & $158.4 \pm 9.7$ & $163.7 \pm 6.3$ & $161.3 \pm 5.9$ & $143.8 \pm 7.6$ & $143.6 \pm 14.5$ & $151.1 \pm 14.6$ \\
\hline ALT (GPT), U/L & $130.7 \pm 3.4$ & $132.2 \pm 4.3$ & $132.9 \pm 4.7$ & $115.6 \pm 3.8$ & $115.4 \pm 10.5$ & $148.6 \pm 12.3$ \\
\hline ALP, U/L & $1,236.8 \pm 95.7$ & $772.9 \pm 53.7$ & $1,343.4 \pm 86.4$ & $973 \pm 91.6$ & $1,203.0 \pm 137.3$ & $829.9 \pm 105.6$ \\
\hline T-bilirubin, mg/dL & $0.19 \pm 0.01$ & $0.19 \pm 0.00$ & $0.18 \pm 0.01$ & $0.19 \pm 0.01$ & $0.18 \pm 0.02$ & $0.19 \pm 0.02$ \\
\hline Glucose, mg/dL & $134.9 \pm 3.3$ & $127.26 \pm 3.1$ & $136.5 \pm 3.5$ & $149 \pm 11.4$ & $123.6 \pm 11.2$ & $138.14 \pm 10.5$ \\
\hline Total protein, $\mathrm{mg} / \mathrm{dL}$ & $6.87 \pm 0.12$ & $6.93 \pm 0.11$ & $6.73 \pm 0.06$ & $6.87 \pm 0.19$ & $6.34 \pm 0.55$ & $7.14 \pm 0.58$ \\
\hline Albumin, g/dL & $2.43 \pm 0.05$ & $2.29 \pm 0.05$ & $2.49 \pm 0.03$ & $2.39 \pm 0.05$ & $2.35 \pm 0.20$ & $2.51 \pm 0.20$ \\
\hline BUN, mg/dL & $13.9 \pm 0.37$ & $11.77 \pm 0.30$ & $12.4 \pm 0.49$ & $12.63 \pm 0.67$ & $11.6 \pm 1.03$ & $12.81 \pm 1.14$ \\
\hline Creatinine, $\mathrm{mg} / \mathrm{dL}$ & $0.56 \pm 0.02$ & $0.54 \pm 0.01$ & $0.60 \pm 0.01$ & $0.54 \pm 0.05$ & $0.56 \pm 0.05$ & $0.57 \pm 0.05$ \\
\hline No. & \multicolumn{2}{|c|}{15} & \multicolumn{2}{|c|}{15} & \multicolumn{2}{|c|}{10} \\
\hline
\end{tabular}

Data are presented as mean \pm standard error of the mean.

BTX, botulinum toxin type A; OP, operation; AST, aspartate transaminase; ALT, alanine transaminase; ALP, alkaline phosphate; T-bilirubin, total bilirubin; BUN, blood urea nitrogen. 
A
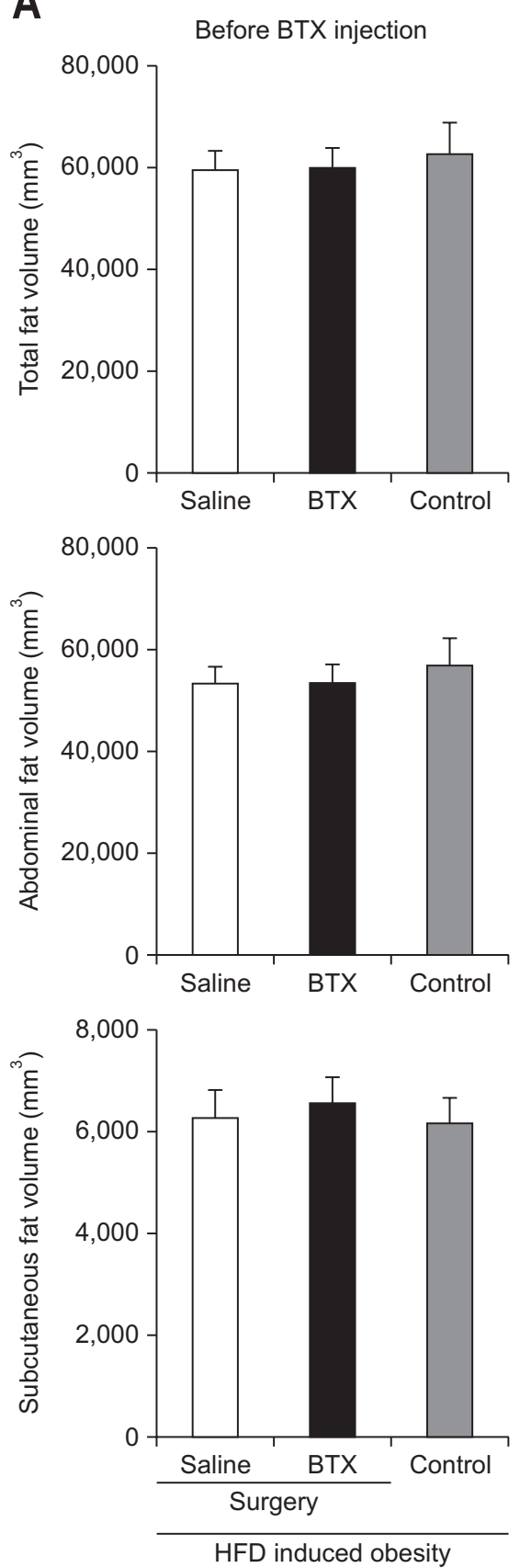

B
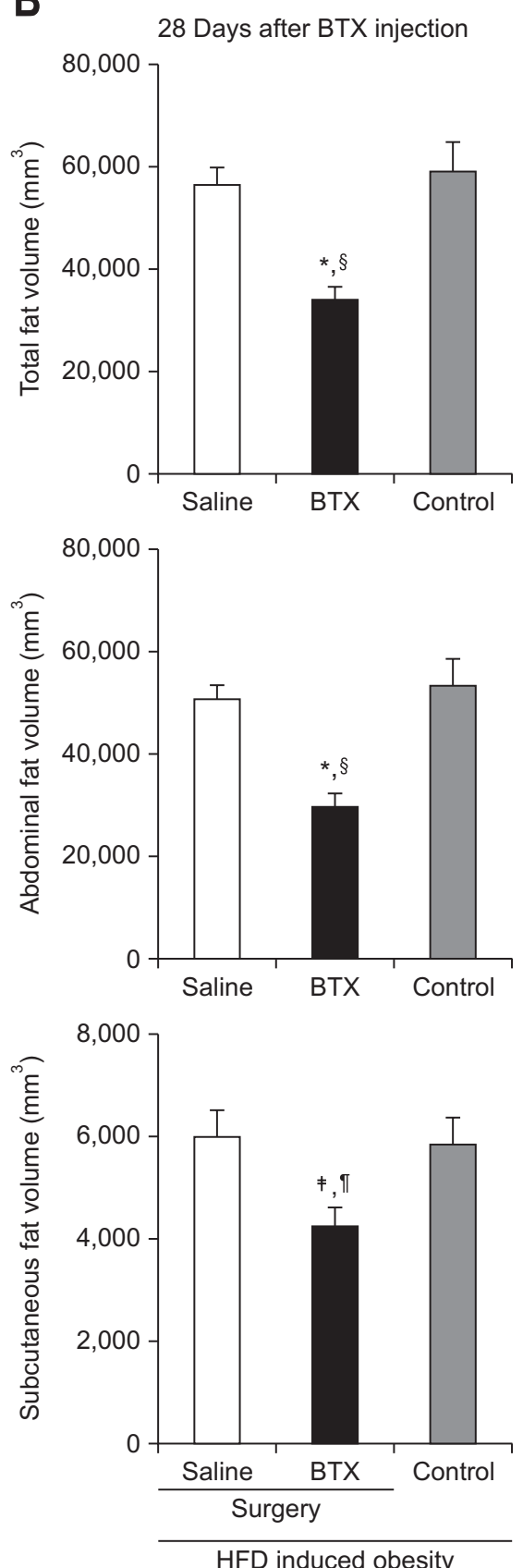

C
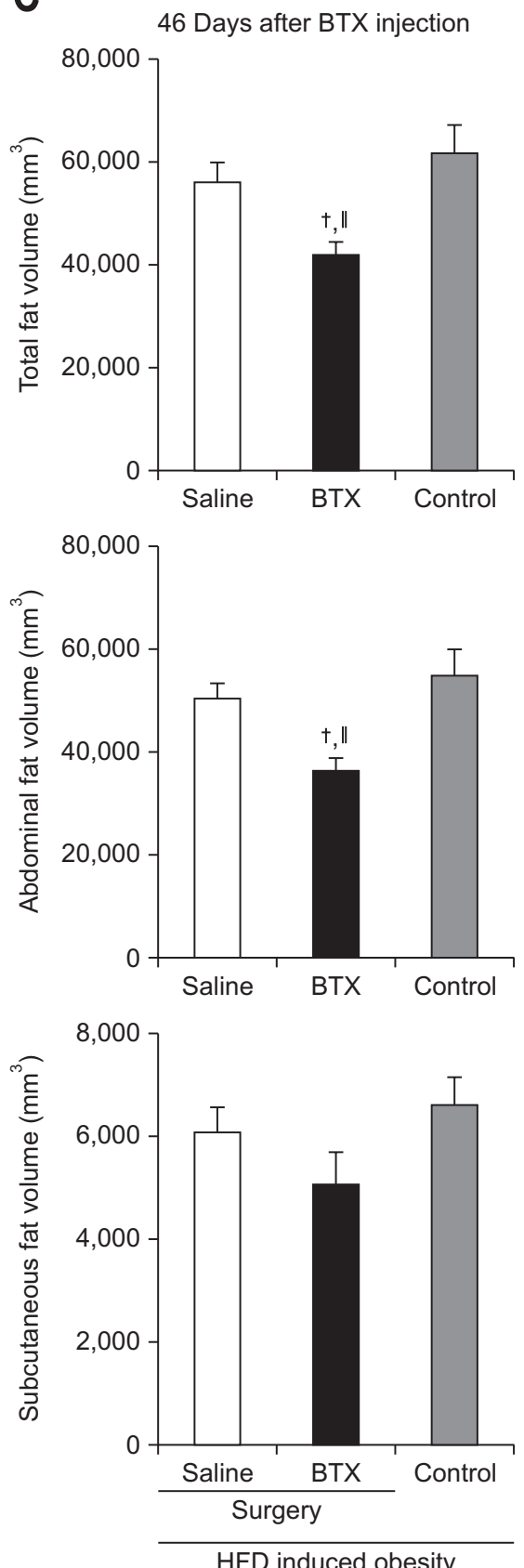

Fig. 6. The effect of botulinum toxin type A (BTX) on body fat volume is shown in the micro-computed tomography images; (A) before BTX injection, (B) 28 days after BTX injection, and (C) 46 days after BTX injection. The reductions tended to be maintained until 46 days after surgery. HFD, high fat diet. A significant difference at ${ }^{*} \mathrm{p}<0.001,{ }^{\dagger} \mathrm{p}<0.01,{ }^{\dagger} \mathrm{p}<0.05$ level compared to the saline group; A significant difference ${ }^{8} \mathrm{p}<0.001$, " $\mathrm{p}<0.01, " \mathrm{p}<0.05$ level compared to the control group.

$\mathrm{p}<0.01)$. These results were similar to those seen in the $a b-$ dominal fat volume (BTX, 29,759.18 $\pm 9,814.69 \mathrm{~mm}^{3}$; control, $\left.53,270.01 \pm 16,631.35 \mathrm{~mm}^{3} ; \mathrm{p}<0.001\right)$. The analysis on the subcutaneous fat volume also showed the same tendency, but the results were without statistical significance.

In the analysis of histologic evaluation, the weights of epididymal fat pads were significantly lower in the BTX group $(14.66 \pm 3.79 \mathrm{~g})$ than in the saline group $(20.32 \pm 5.88 \mathrm{~g}, \mathrm{p}<0.05)$. In addition, the weight of the perinephric fat pad was also lower in the BTX group than in the saline $(38.0 \pm 9.58 \mathrm{~g}, \mathrm{p}<0.01)$ and control groups $(47.73 \pm 13.97 \mathrm{~g}, \mathrm{p}<0.001)$ (Fig. 7).

\section{Effects on BTX on hepatic lipid profiles and hepatic histology}

The weights of the livers were not significantly different among the groups, and the hepatic total cholesterol level was also similar between the groups. However, the hepatic TG level was significantly lower in the BTX group compared 


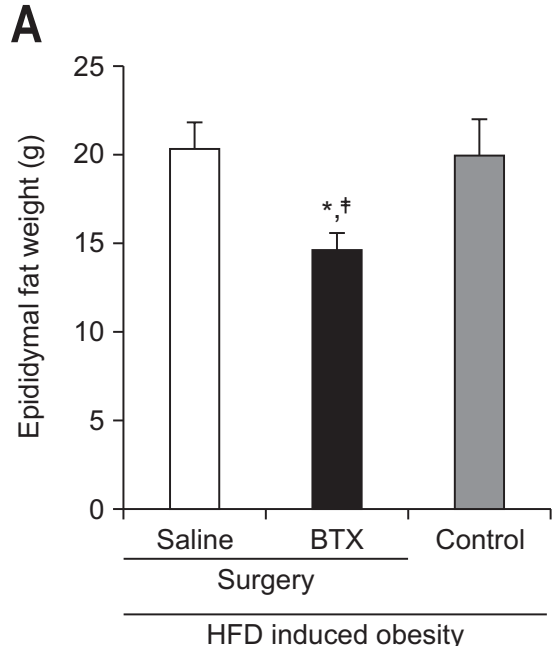

HFD induced obesity
B

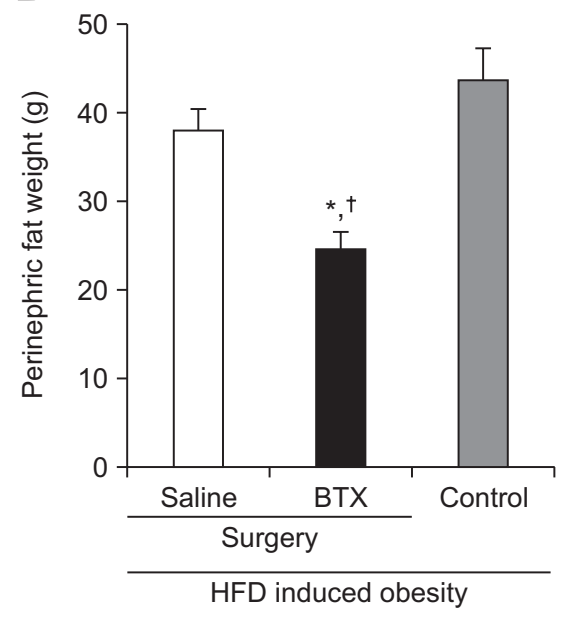

Fig. 7. Effects of botulinum toxin type A (BTX) on reducing epididymal (A) and perinephric fat (B). The weights of the epididymal fat pad and perinephric fat pad were lower in the BTX group than in the other groups.

HFD, high fat diet. *A significant difference at $\mathrm{p}<0.01$ level compared to the saline group; A significant difference ${ }^{\dagger} \mathrm{p}<0.01,{ }^{\ddagger} \mathrm{p}<0.05$ level compared to the control group.
A

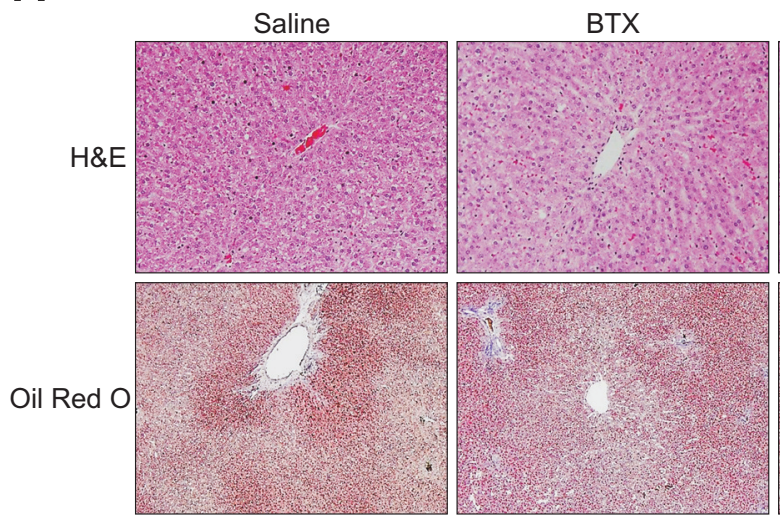

B

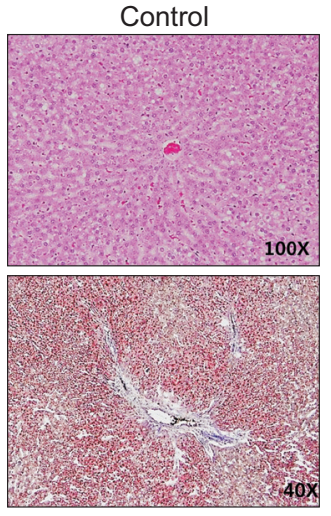

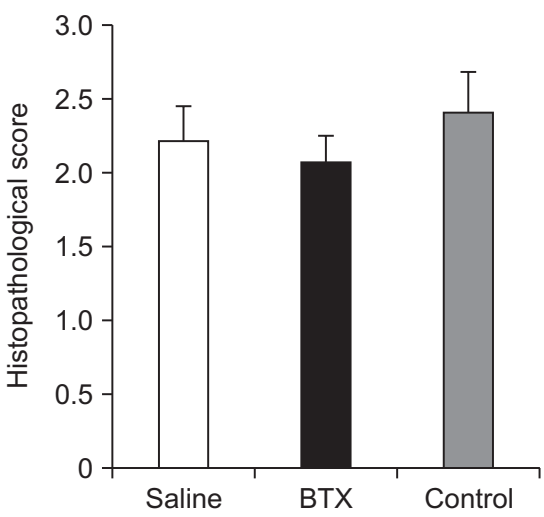

Fig. 8. Effects of botulinum toxin type A (BTX) on reducing the hepatic lipid levels. (A) Oil Red 0 staining in liver tissue sections from the three groups at 6 weeks after surgery. (B) The histopathological scores of fat deposits calculated from the Oil Red 0 staining were not significantly different among the three groups.

with the saline group. The hepatic TG level of the saline group $(14.96 \pm 4.40)$ was about 1.3 times higher than that of the BTX group $(11.78 \pm 2.51, \mathrm{p}<0.05)$. The result of H\&E staining of liver tissues showed that there were extensive cytoplasmic fat deposition vacuoles in all obese rats, and that there was no difference of fat accumulation between the groups. The result of Oil Red 0 staining of liver tissues demonstrated diffuse oil droplet accumulation, and a difference was to an extent detected in all three groups (Fig. 8). However, the hepatic fat area calculated by Oil Red 0 staining was not significantly different in the BTX group compared with the other groups.

\section{DISCUSSION}

Obesity strongly affects the gastrointestinal function by direct and indirect mechanisms, resulting in a reinforcement of the ability of patients to increase food intake progressively through an inhibition of satiety signals. ${ }^{16}$ Accelerated gastric emptying, and thereby inducing overeating, is generally accepted as the main mechanism that contributes to the development of obesity. ${ }^{17}$ Cardoso-Júnior et al. ${ }^{18}$ demonstrated that morbidly obese patients show enhanced gastric emptying of solids, with a direct correlation with body mass index. Currently, bariatric operation and a pacemaker in the gastric wall are two modalities used to control the gastric emptying as a treatment for obesity; there are previous reports that show results from these methods. ${ }^{19,20}$

Based on the same principle, the use of gastric BTX injection could offer alternative options as the treatment for obesity. BTX type A is highly selective for cholinergic cell and it inhibits the release of acetylcholine at the neuromuscular junction, resulting in subsequent local muscle paralysis. ${ }^{21}$ The acetylcholine-mediated gastric antral contraction is essential step for food passage into the duodenum, with fragmentation of solid nutrients and differentiation of solid-liquid components, and sequential fundal relaxation. Accordingly, BTX that was injected into the antral muscular wall can, theoretically, selectively affected gas- 
tric emptying by reducing acetylcholine-mediated gastric contraction. Another theory for weight reduction by BTX injection is inducing early satiety. The sense of fullness is created by the hypothalamic ventral medial nuclei stimulation as the stomach gets full and stretches. Therefore, gastric BTX injection can lead to early satiety since the tension in the stomach to be longer by delaying gastric emptying, and the desire to eat would be decreased.

In the current study, gastric BTX injections were clearly demonstrated to be effective in the reduction of body weight and in the inhibition of gastric emptying. One week after operation, the postoperative body weight of the BTX group was significantly reduced than that of the saline and control groups. The reduction rates for the BTX, and saline groups were 9.4\%, and 0.25\%, and control group rather gained body weight as much as $2.1 \%$. With respect to gastric emptying, the gastric emptying time in the BTX group was significantly delayed after the operation (1.7 \pm 0.6 minutes vs $2.6 \pm 0.9$ minutes). Therefore, we estimated that our results provide firm evidence that gastric BTX injection may be a promising treatment modality for obesity. In an attempt to verify the effect of BTX on weight reduction, the weight of epididymal and perinephric fats after necropsy and fat volume by micro-CT were measured. The weights of fat pads and fat volumes were significantly lower in the BTX group than in the saline and control groups. These results suggest that BTX injection may be attributable to decreased lipid accumulation in the adipose tissue by BTX's antiobesity effect.

Further safety consideration should be addressed concerning the possible systemic toxic effect from using BTX in the stomach. In the current study, we performed a hematologic examination in order to identify subclinical signs of distant spread of BTX post an intragastric BTX treatment. Injection of BTX is generally well-tolerated without significant adverse events. Moreover, BTX injection did not show any renal or hepatic toxicity.

Although BTX injection reduced food intake and weight gain more profoundly in obese rats, we found that this treatment reduced the progression of nonalcoholic fatty liver disease in obese rats, with an antisteatotic activity. Liver steatosis is a wellknown pathology in severely obese patients and is especially associated with visceral adiposity and diabetes. Our understanding of the histologic mechanism of nonalcoholic fatty liver disease has not been fully understood thus far. However, it is believed that an increase in the levels of hepatic TG causes hepatic steatosis. ${ }^{22}$ The nonesterified fatty acids that are incorporated into the TG within the liver-so called "de novo lipogenesis"-plays a substantial role in the pathogenesis of nonalcoholic fatty liver disease, accounting for $26 \%$ of hepatic TGs in humans. ${ }^{23}$ In this study, gastric antral injection of BTX in rats resulted in a significant decrease in the hepatic TG level compared with the saline group. In addition, circulating levels of total cholesterol and LDL in the BTX group were significantly lower than those in the control group. We believed that this antisteatotic activity, including a decrease of the hepatic TG accumulation in the BTX group, may be associated with weight reduction via a strong inhibitory effect of BTX on gastric emptying. The effect of weight loss on hepatic TG reduction was demonstrated in previous study. Browning et al. ${ }^{24}$ studied with eighteen nonalcoholic fatty liver disease patients to determine the effectiveness of 2 weeks of dietary restriction at reducing hepatic TGs. In the results, all patients were achieved weight loss in 2 weeks and liver TG decreased significantly ( $\mathrm{p}<0.001)$. Therefore, we suggest that gastric BTX injection be used as a treatment for nonalcoholic fatty liver diseases, as well as obesity; however, it is important to note that further studies are encouraged to further determine the effects of gastric BTX injection on hepatic steatosis.

The primary limitation of this study is the short observation period. BTX is well known to have a long-lasting effect; but its usual significant response lasts only 1 to several months. Therefore, it is uncertain whether the weight reduction could be sustained for a long period. In current study, weight reduction curves in the BTX group continued for 7 weeks after gastric BTX injection, however, the weight was gradually recovered during the study period. Therefore, the effect of BTX on weight reduction might not be maintained over 7 weeks in our opinion. To achieve the effect more effectively with BTX, additional gastric injection would be required after some time has passed. However, the maintenance period of BTX effect in stomach is not well studied yet. Further studies are necessary to confirm this issue.

In conclusion, gastric BTX injection has a beneficial effectreduction of body weight, adipose tissue weight, and lipid level in the serum and liver in obese rats. Therefore, gastric BTX injection has the potential to be an effective therapeutic strategy with no obvious toxicity against obesity-related diseases.

\section{CONFLICTS OF INTEREST}

No potential conflict of interest relevant to this article was reported.

\section{ACKNOWLEDGEMENTS}

This research was supported by the Inha University Hospital Grant. All endoscopic procedures were performed at the large animal center of the national Center of Efficacy Evaluation for the Development of Health Products Targeting Digestive Disorders.

Author contribution: J.S.P. and H.M.Z. conception and design, collection and assembly of data, analysis and interpretation of the data, drafting of the article, provision of study materials, administrative and technical or logistic support. C.S.K. administrative and technical support for the animal experiments. S.J. administrative and technical or logistic support for the animal 
experiments. D.H.L. conception and design, provision of study materials, critical revision of the article for important intellectual content, and final approval of the article.

\section{REFERENCES}

1. Wang YC, McPherson K, Marsh T, Gortmaker SL, Brown M. Health and economic burden of the projected obesity trends in the USA and the UK. Lancet 2011;378:815-825.

2. Swinburn BA, Sacks G, Hall KD, et al. The global obesity pandemic: shaped by global drivers and local environments. Lancet 2011;378:804-814.

3. Bays HE. Current and investigational antiobesity agents and obesity therapeutic treatment targets. Obes Res 2004;12:1197-1211.

4. Mun EC, Blackburn GL, Matthews JB. Current status of medical and surgical therapy for obesity. Gastroenterology 2001;120:669681.

5. Foschi D, Corsi F, Pisoni L, et al. Plasma cholecystokinin levels after vertical banded gastroplasty: effects of an acidified meal. Obes Surg 2004;14:644-647.

6. Cummings DE, Weigle DS, Frayo RS, et al. Plasma ghrelin levels after diet-induced weight loss or gastric bypass surgery. N Engl J Med 2002;346:1623-1630.

7. Espinet-Coll E, Nebreda-Durán J, Gómez-Valero JA, et al. Current endoscopic techniques in the treatment of obesity. Rev Esp Enferm Dig 2012;104:72-87.

8. Albani G, Petroni ML, Mauro A, et al. Safety and efficacy of therapy with botulinum toxin in obesity: a pilot study. J Gastroenterol 2005;40:833-835.

9. Gui D, Mingrone G, Valenza V, et al. Effect of botulinum toxin antral injection on gastric emptying and weight reduction in obese patients: a pilot study. Aliment Pharmacol Ther 2006;23:675-680.

10. Coskun H, Duran Y, Dilege E, Mihmanli M, Seymen H, Demirkol MO. Effect on gastric emptying and weight reduction of botulinum toxin-A injection into the gastric antral layer: an experimental study in the obese rat model. Obes Surg 2005;15:1137-1143.

11. Júnior AC, Savassi-Rocha PR, Coelho LG, et al. Botulinum A toxin injected into the gastric wall for the treatment of class III obesity: a pilot study. Obes Surg 2006;16:335-343.

12. Kim CS, Jang WS, Son IP, et al. Electrophysiological study for comparing the effect of biological activity between type A botulinum toxins in rat gastrocnemius muscle. Hum Exp Toxicol
2013;32:914-920.

13. Nam HS, Park YG, Paik NJ, et al. Efficacy and safety of NABOTA in post-stroke upper limb spasticity: a phase 3 multicenter, doubleblinded, randomized controlled trial. J Neurol Sci 2015;357:192197.

14. Won CH, Kim HK, Kim BJ, et al. Comparative trial of a novel botulinum neurotoxin type A versus onabotulinumtoxinA in the treatment of glabellar lines: a multicenter, randomized, doubleblind, active-controlled study. Int J Dermatol 2015;54:227-234.

15. Canny J. A computational approach to edge detection. IEEE Trans Pattern Anal Mach Intell 1986;8:679-698.

16. Abraham S, Kellow JE. Do the digestive tract symptoms in eating disorder patients represent functional gastrointestinal disorders? BMC Gastroenterol 2013;13:38.

17. Xing J, Chen JD. Alterations of gastrointestinal motility in obesity. Obes Res 2004;12:1723-1732.

18. Cardoso-Júnior A, Coelho LG, Savassi-Rocha PR, et al. Gastric emptying of solids and semi-solids in morbidly obese and nonobese subjects: an assessment using the 13C-octanoic acid and 13C-acetic acid breath tests. Obes Surg 2007;17:236-241.

19. Cigaina V. Long-term follow-up of gastric stimulation for obesity: the Mestre 8-year experience. Obes Surg 2004;14 Suppl 1:S14-S22.

20. Chevallier JM, Zinzindohoué F, Douard R, et al. Complications after laparoscopic adjustable gastric banding for morbid obesity: experience with 1,000 patients over 7 years. Obes Surg 2004;14:407414.

21. James AN, Ryan JP, Parkman HP. Inhibitory effects of botulinum toxin on pyloric and antral smooth muscle. Am J Physiol Gastrointest Liver Physiol 2003;285:G291-G297.

22. Adams LA, Sanderson S, Lindor KD, Angulo P. The histological course of nonalcoholic fatty liver disease: a longitudinal study of 103 patients with sequential liver biopsies. J Hepatol 2005;42:132138.

23. Donnelly KL, Smith CI, Schwarzenberg SJ, Jessurun J, Boldt MD, Parks EJ. Sources of fatty acids stored in liver and secreted via lipoproteins in patients with nonalcoholic fatty liver disease. J Clin Invest 2005;115:1343-1351.

24. Browning JD, Baker JA, Rogers T, Davis J, Satapati S, Burgess SC. Short-term weight loss and hepatic triglyceride reduction: evidence of a metabolic advantage with dietary carbohydrate restriction. Am J Clin Nutr 2011;93:1048-1052. 